ISSN 1112-9867

http://www.jfas.info

\title{
ABSCISIC ACID EFFECTS ON WATER AND PHOTOSYNTHETIC CHARACTERISTICS OF TWO ECOTYPES OF Atriplex halimus L.
}

\author{
Y. Bidai*, A. Achour and M. Belkhodja \\ Laboratoire de Physiologie Végétale, Département de Biologie, Faculté des Sciences \\ de la Nature et de la Vie, Université Oran 1 Ahmed Ben Bella, Oran, Algérie
}

Received: 03 February 2016 / Accepted: 21 April 2016 / Published online: 01 May 2016

\begin{abstract}
The aim of this study is to compare the water and photosynthetic characteristics of two xerophilic ecotypes of Atriplex halimus (L.). Seeds collected from two different sites Djelfa and Oran are germinated in controlled greenhouse. After 6 months, the plantlets were treated 21 days with increasing concentrations of abscisic acid (0M, 10-6 M and 10-3 M). The results show that ecotype of Djelfa reduced water loss through transpiration because of high stomatal resistance. Consequently the content of chlorophyll a and b decrease significantly compared to Oran ecotype which show an increase of the osmotic potential and relative water content. Osmotic adjustment to reduce dehydration and maintain a good photosynthesis seems efficient in Oran ecotype.
\end{abstract}

Keywords: Phytohormone; drought; xerophyte; water status; stomatal resistance; chlorophyll.

Author Correspondence, e-mail: ybidai@yahoo.fr

doi: http://dx.doi.org/10.4314/jfas.v8i2.19

\section{INTRODUCTION}

Dans les écosystèmes arides, la sécheresse est un facteur abiotique affectant les processus physiologiques [1] et hormonaux [2] des plantes induisant la réduction de la croissance, du 
développement et du rendement des cultures $[3,4]$. Face à cette contrainte hydrique, les plantes mettent en place différentes stratégies d'adaptation en particulier, l'esquive, l'évitement ou la tolérance [5,6]. La plupart de ses stratégies s'expriment par des réponses diverses notamment hydrique et physiologique chez de nombreuses espèces sous l'action de facteurs hormonaux comme l'acide abscissique (ABA) [7, 8]. En effet, diverses contraintes induisent la synthèse de l'ABA, considéré aujourd'hui comme une hormone de stress [9, 10,11]. Lors d'un stress hydrique, l'ABA formé dans les racines est transporté dans la partie aérienne $[12,13]$ induisant la fermeture des stomates $[14,15]$. Cette fermeture stomatique réduit ainsi les pertes en eau par transpiration, maintient la croissance des pousses et favorise la croissance des racines $[9,16]$. Ce mécanisme d'évitement permet par ailleurs de maintenir un niveau d'hydratation ce qui préserve un potentiel hydrique élevé dans les tissus des plantes pendant la contrainte $[17,18]$. En conséquence, la fermeture stomatique conduira à la limitation de la transpiration [19], à un ralentissement de la photosynthèse [20] et plus tard à la réduction de la surface foliaire $[21,20]$. L'identification de génotypes capables de maintenir une photosynthèse active et de produire un rendement en biomasse optimal sous contrainte hydrique, repose sur une réduction des mécanismes d'évitement induits par l'ABA. L'utilisation de l'ABA dans un programme de sélection permettrait la liaison entre un critère de sélection analytique (réactivité vis-à-vis de l'ABA ou aptitude à accumuler l'ABA) et des processus physiologiques globaux, comme les régulations stomatiques, le statut hydrique et l'activité photosynthétique [22].

L'objectif de notre travail vise à examiner les réponses de deux écotypes d'Atriplex halimus L. soumises à un régime hormonal de l'ABA. Pour apprécier l'action hormonale sur le comportement des plantes juvéniles, nous analysons d'abord les paramètres hydriques comme les pertes en eau par transpiration (RWL), la teneur relative en eau (RWC) et le potentiel osmotique ( $\Psi$ o), puis les paramètres physiologiques comme la résistance stomatique (RS) et la photosynthèse à travers une quantification des pigments chlorophylliens (chl a et chl b).

\section{MATERIEL ET METHODES}

\subsection{Matériel végétal}

Un essai a été conduit en serre contrôlée à l'université d'Oran sur des graines d'Atriplex halimus L. récoltées de deux sites différents en Algérie: campus de l'université, une zone 
littorale à climat méditerranéen avec environ 420 mm de pluie par an et El-Mesrane (Djelfa), une zone intérieure à climat semi aride recevant en moyenne $350 \mathrm{~mm}$ de pluie par an.

\subsection{Culture et application du traitement}

Les graines sont débarrassées de leurs bractées puis mises à germer. Les plantules âgées de six mois sont ensuite soumises à un traitement de trois semaines à l'ABA exogène dans des conditions d'arrosages convenables. Une pulvérisation quotidienne de l'acide abscissique (2cis, 4-trans-Abscisic acid, 98\%) est appliquée sur la partie aérienne des plantes. Les plantes témoins sont pulvérisées seulement à l'eau distillée. L'essai a été installé suivant un dispositif randomisé à deux facteurs et à cinq répétitions (30 plantules par écotype). Le facteur principal concerne l'ABA à trois concentrations, $0 \mathrm{M}, 10^{-6} \mathrm{M}$ et $10^{-4} \mathrm{M}$. Le deuxième facteur est représenté par les deux écotypes (Oran et Djelfa).

\subsection{Paramètres analysés}

\subsubsection{Taux de déperdition en eau}

Le taux de déperdition en eau (Rate Water Loss : RWL) par transpiration est évalué à partir des feuilles détachées après 30 mn, 60 mn et 120 mn d'exposition selon la méthode de [23].

\subsubsection{Résistance stomatique (RS)}

La résistance stomatique $\left(\mathrm{cm} . \mathrm{s}^{-1}\right)$ qui traduit le degré d'ouverture des stomates, est mesurée par la méthode porométrique à diffusion de vapeur d'eau.

\subsubsection{Teneur en eau}

La teneur relative en eau (Relative Water Content : RWC) est établie par la méthode de [24]. $\mathrm{RWC}(\%)=[(\mathrm{PF}-\mathrm{PS}) /(\mathrm{PT}-\mathrm{PS})] \mathrm{X} 100$

PF: poids frais, PT: poids à la turgescence, PS: poids sec

\subsubsection{Potentiel osmotique ( $\Psi 0)$}

L'estimation de ce paramètre est obtenue à l'aide d'un osmomètre de type Loser MeBtechnik sur des extraits de feuilles. L'abaissement du point de congélation par rapport à l'eau distillée donne une mesure directe de la concentration molaire en mosmoles. $\mathrm{Kg}^{-1} \mathrm{H}_{2} \mathrm{O}$

\subsubsection{Analyse des pigments chlorophylliens}

L'extraction et le dosage des pigments photosynthétiques, chlorophylle a et chlorophylle b se font selon le protocole de [25]. La lecture est faite au spectrophotomètre à UV visible à deux 
longueurs d'onde $645 \mathrm{~nm}$ et $663 \mathrm{~nm}$. Les teneurs en chlorophylles, exprimées en $\mathrm{mg}^{\mathrm{m}} \mathrm{ml}^{-1}$, sont calculées selon les formules suivantes:

Chl a $\left(\mathrm{mg}^{\left.-\mathrm{ml}^{-1}\right)}=12,7 \times \mathrm{DO}_{663}-2,69 \times \mathrm{DO}_{645}\right.$

Chl b $\left(\mathrm{mg} \mathrm{ml}^{-1}\right)=22,9 \times \mathrm{DO}_{645}-4,68 \times \mathrm{DO}_{663}$

\subsubsection{Analyses statistiques}

Les résultats obtenus sont analysés à l'aide du logiciel SPSS (version 17.0). Une analyse de variance multivariée (MANOVA) est effectuée pour évaluer l'effet de l'acide abscissique sur quelques paramètres physio-biochimiques (résistance stomatique, potentiel osmotique, teneur relative en eau et la teneur des chlorophylles a et b) chez deux écotypes d'Atriplex halimus selon le critère de la trace de Pillai. Une analyse de variance à un seul facteur (ANOVA) est ensuite menée pour déterminer l'effet des traitements sur chaque paramètre, puis les moyennes sont comparées par le test LSD $(\alpha<0.05)$. De même les coefficients de Corrélation de Pearson ont été calculés pour déterminer les relations entre les variables retenues.

\section{RESULTATS}

\subsection{Déperdition en eau}

L'analyse de la variance (Tableau 1) montre que l'ABA a un effet significatif sur la perte d'eau par transpiration $(\mathrm{F}=514,75 ; \mathrm{p}=0,000$ à $\alpha<0.05)$. Cette dernière mesurée en fonction de la durée est inversement proportionnelle à la concentration de l'ABA. Une corrélation négative hautement significative entre l'ABA et la transpiration est notée d'une part $(\mathrm{r}=0,435, \mathrm{p}<0.01)$ et d'autre part entre la transpiration et la durée $(\mathrm{r}=0,763, \mathrm{p}<0.01)$ (Tableau 2).

Tableau 1. RWL : tests des effets inter-sujets

\begin{tabular}{|l|l|l|l|l|l|l|}
\hline Source & $\begin{array}{l}\text { Variable } \\
\text { dépendante }\end{array}$ & $\begin{array}{l}\text { Somme des carrés } \\
\text { de type III }\end{array}$ & ddl & $\begin{array}{l}\text { Moyenne } \\
\text { des carrés }\end{array}$ & F & Sig. \\
\hline ABA & RWL &, 017 & 2 &, 009 & 514,758 &, 000 \\
\hline Écotype & RWL &, 001 & 1 &, 001 & 31,376 &, 000 \\
\hline Durée & RWL &, 041 & 2 &, 021 & 1218,022 &, 000 \\
\hline Écotype*ABA* Durée & RWL &, 009 & 12 &, 001 & 46,508 &, 000 \\
\hline
\end{tabular}


Tableau 2. Relation entre l'ABA, l'écotype, la durée et la RWL (corrélation de Pearson)

\begin{tabular}{|l|l|l|l|l|}
\hline Variables & Ecotype & ABA & Durée & RWL \\
\hline Ecotype & 1 & & & \\
\hline ABA &,- 013 & 1 & & \\
\hline Durée &,- 013 &, 016 & 1 & \\
\hline RWL &, 101 &,$- 435^{* *}$ &,$- 763^{* *}$ & 1 \\
\hline
\end{tabular}

**: Significatif à $\mathrm{p}<0.01$

La Figure 1 montre que chez les deux écotypes Oran (A) et Djelfa (B), la transpiration diminue après une durée de 30 à $60 \mathrm{mn}$ sous le traitement $\mathrm{ABA}$ à $10^{-4} \mathrm{M}$, ce qui traduit une fermeture précoce des stomates par rapport à celle du témoin.

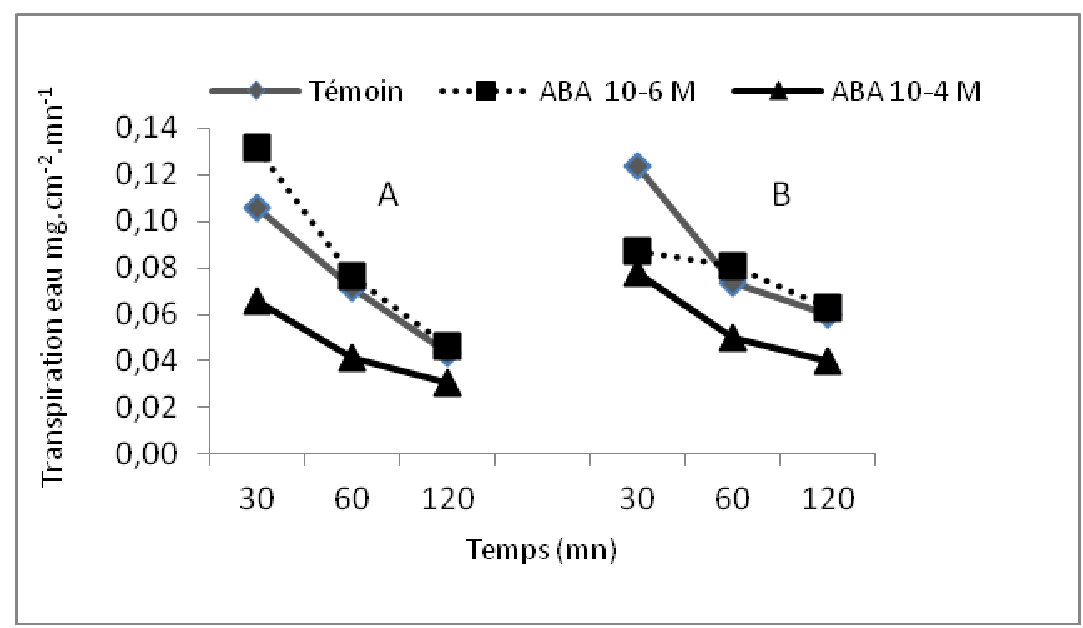

Fig.1. Évolution en fonction du temps de la transpiration (RWL) des feuilles de deux écotypes, Oran (A) et Djelfa (B) après 21 jours de traitement à l'acide abscissique exogène

Pour l'écotype d'Oran, les RWL enregistrées au bout de $30 \mathrm{mn}$ varient de $0.11 \mathrm{mg} . \mathrm{cm}^{-}$ ${ }^{2} \cdot \mathrm{mn}^{-1}$ pour le témoin à $0.07 \mathrm{mg} \cdot \mathrm{cm}^{-2} \cdot \mathrm{mn}^{-1}$ pour les feuilles des plantes recevant $10^{-4} \mathrm{M}$ ABA. Jusqu'à $120 \mathrm{mn}$ d'observation, la transpiration poursuit son ralentissement pour atteindre un RWL de $0.04 \mathrm{mg} \cdot \mathrm{cm}^{-2} \cdot \mathrm{mn}^{-1}$ et $0.03 \mathrm{mg} \cdot \mathrm{cm}^{-2} \cdot \mathrm{mn}^{-1}$ respectivement pour le témoin et le traité à $10^{-4} \mathrm{M} \mathrm{ABA}$.

Pour l'écotype de Djelfa, le RWL évolue à la baisse sous la concentration d'ABA $10^{-4} \mathrm{M}$ dans le même sens que celui signalé pour l'écotype d'Oran. Il faut remarquer que les pertes en eau par transpiration évoluent lentement comparativement toujours à l'écotype d'Oran. Sous le traitement à $10^{-6} \mathrm{M} \mathrm{ABA}$, à $30 \mathrm{mn}$, les stomates des feuilles des plantes de l'écotype d'Oran 
suivent un rythme de transpiration plus rapide que ceux de l'écotype de Djelfa ; les valeurs du RWL varient respectivement de 0.13 à $0.09 \mathrm{mg} \cdot \mathrm{cm}^{-2} \cdot \mathrm{mn}^{-1}$. Par contre, la réaction des plantes ne se manifeste qu'après un délai de $60 \mathrm{mn}$ si bien que les stomates des feuilles des plantes des deux écotypes ralentissent leur rythme transpiratoire pour atteindre des RWL respectifs identiques $\left(0.08 \mathrm{mg} \cdot \mathrm{cm}^{-2} \cdot \mathrm{mn}^{-1}\right)$.

Selon les résultats de l'analyse statistique, il apparaît que les variations de la RWL sont dépendantes du génotype $(\mathrm{F}=31,37 ; \mathrm{p}=0,000$ à $\alpha<0.05)$, de la durée $(\mathrm{F}=1218,02 ; \mathrm{p}=0,000$ à $\alpha<0.05)$ et enfin de l'interaction " ABA x écotype x Durée" ( $F=46,50 ; p=0,000$ à $\alpha<0.05)$ (Tableau 1).

\subsection{Résistance stomatique}

Les stomates réagissent aux conditions du traitement à l'ABA, en fonction de la concentration de cette hormone. La RS des feuilles affiche des valeurs croissantes chez les deux écotypes (Figure 2).

Néanmoins, ce paramètre évolue de manière très lente pour l'écotype d'Oran ; dans ce cas, la RS passe de $0.034 \mathrm{~cm} . \mathrm{s}^{-1}$ chez le témoin à des valeurs variant entre $0.044 \mathrm{~cm} . \mathrm{s}^{-1}$ pour les feuilles des plantes stressées à $10^{-6} \mathrm{M}$ ABA et elle double sous $1^{1} \mathrm{ABA}$ à $10^{-4} \mathrm{M}\left(0.64 \mathrm{~cm} \cdot \mathrm{s}^{-1}\right)$. Les plantes de l'écotype de Djelfa s'avèrent plus résistantes pour le maintien de l'hydratation cellulaire. la RS varie avec des valeurs plus élevées, deux fois plus sous le traitement $10^{-4} \mathrm{M}$ ABA par rapport à la RS des feuilles de l'écotype d'Oran sous le même traitement $(0,145$ $\mathrm{cm} . \mathrm{s}^{-1}$ contre $0.063 \mathrm{~cm} \cdot \mathrm{s}^{-1}$ ). Sous le traitement $\mathrm{ABA}$ à $10^{-6} \mathrm{M}$, la RS représente une valeur trois fois plus celle enregistrée pour l'écotype d'Oran par rapport à celle de l'écotype de Djelfa $\left(0.12 \mathrm{~cm} . \mathrm{s}^{-1}\right.$ contre $\left.0.04 \mathrm{~cm} \cdot \mathrm{s}^{-1}\right)$. 


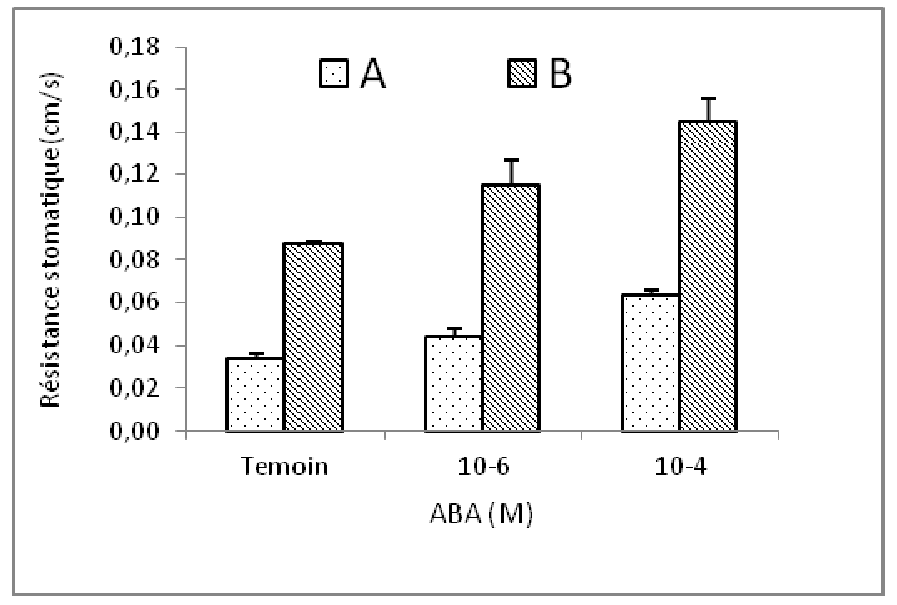

Fig.2. Variation de la résistance stomatique des feuilles de deux écotypes, Oran (A) et Djelfa (B) après 21 jours de traitement à l'acide abscissique exogène

L'analyse statistique de ce paramètre (Tableau 3) révèle un effet hautement significatif du traitement $\mathrm{ABA}(\mathrm{F}=101,516 ; \mathrm{p}=0,000$ à $\alpha<0.05)$, de l'écotype $(\mathrm{F}=757,485 ; \mathrm{p}=0,000$ à $\alpha$ $<0.05)$ et de l'interaction " ABA x écotype" $(\mathrm{F}=10,799 ; \mathrm{p}=0,000$ à $\alpha<0.05)$ sur la résistance stomatique. Cela signifie que l'acide abscissique a un effet sur les mouvements stomatiques et que l'expression de ce caractère reste fortement influencée par la variabilité génotypique et par l'interaction entre les deux facteurs.

Par ailleurs, la résistance stomatique est positivement corrélée avec le traitement hormonal à l'ABA $(\mathrm{r}=0,448, \mathrm{p}<0.013)($ Tableau 4).

Tableau 3. RWC, RS, $\Psi_{0}$, chl a et chl b: tests des effets inter-sujets

\begin{tabular}{|c|c|c|c|c|c|c|}
\hline Source & $\begin{array}{c}\text { Variable } \\
\text { dépendante }\end{array}$ & $\begin{array}{c}\text { Somme des carrés } \\
\text { de type III }\end{array}$ & $\mathrm{ddl}$ & $\begin{array}{l}\text { Moyenne } \\
\text { des carrés }\end{array}$ & $\mathrm{F}$ & Sig. \\
\hline \multirow[t]{5}{*}{$\mathrm{ABA}$} & RWC & 1250,931 & 2 & 625,466 & 88,671 & ,000 \\
\hline & RS & ,010 & 2 & ,005 & 101,516 &, 000 \\
\hline & $\Psi о$ & 10629,206 & 2 & 5314,603 & 1353,523 & ,000 \\
\hline & chl a & 6000,573 & 2 & 3000,287 & 22,730 & ,000 \\
\hline & chl b & 2327,108 & 2 & 1163,554 & 14,693 &, 000 \\
\hline \multirow[t]{4}{*}{ Ecotype } & RWC & 6,665 & 1 & 6,665 & ,945 & ,341 \\
\hline & RS & ,036 & 1 & ,036 & 757,485 & ,000 \\
\hline & $\Psi$ \%o & 53427,479 & 1 & 53427,479 & 13606,913 & ,000 \\
\hline & Chl a & 95530,776 & 1 & 95530,776 & 723,728 &, 000 \\
\hline
\end{tabular}




\begin{tabular}{|c|c|c|c|c|c|c|}
\hline & Chl b & 5064,477 & 1 & 5064,477 & 63,951 &, $\mathbf{0 0 0}$ \\
\hline ABA*Ecotype & RWC & 1887,539 & 2 & 943,769 & 133,796 & $\mathbf{0 0 0}$ \\
\cline { 2 - 7 } & RS &, 001 & 2 &, 001 & 10,799 & $\mathbf{, 0 0 0}$ \\
\cline { 2 - 7 } & To & 126994,497 & 2 & 63497,249 & 16171,483 & $\mathbf{0 0 0}$ \\
\cline { 2 - 7 } & Chl a & 6678,103 & 2 & 3339,051 & 25,296 & $\mathbf{0 0 0}$ \\
\cline { 2 - 7 } & Chl b & 1242,132 & 2 & 621,066 & 7,842 & $\mathbf{0 0 2}$ \\
\hline
\end{tabular}

Tableau 4. Corrélation entre l'acide abscissique, l'écotype et les paramètres étudiés

\begin{tabular}{|c|c|c|c|c|c|c|c|}
\hline Variables & Ecotype & $\mathrm{ABA}$ & RWC & $\mathrm{RS}$ & $\Psi о$ & chl a & chl b \\
\hline Ecotype & 1 & & & & & & \\
\hline $\mathrm{ABA}$ & ,028 & 1 & & & & & \\
\hline RWC & ,054 &,- 277 & 1 & & & & \\
\hline $\mathrm{RS}$ & ,868** & ,448* &,- 203 & 1 & & & \\
\hline$\Psi$ о &, $529 * *$ &,- 198 &,$- 582 * *$ & ,460* & 1 & & \\
\hline chl a &,$- 926 * *$ &,- 040 &,- 037 &,$- 863 * *$ &,$- 550 * *$ & 1 & \\
\hline chl b &,$- 693 * *$ &,$- 444 *$ &,- 173 &,$- 812 * *$ &,- 027 &, $679 * *$ & 1 \\
\hline
\end{tabular}

** : Significatif à $\mathrm{p}<0.01$

* : Significatif à $\mathrm{p}<0.05$

\subsection{Influence de l'ABA exogène sur le $\Psi 0$ et la RWC}

Les variations du potentiel osmotique chez les deux écotypes au cours du traitement hormonal et celles de la teneur en eau sont présentées dans la Figure 3. L'ABA exerce un effet hautement significatif sur l'expression de ces deux paramètres.

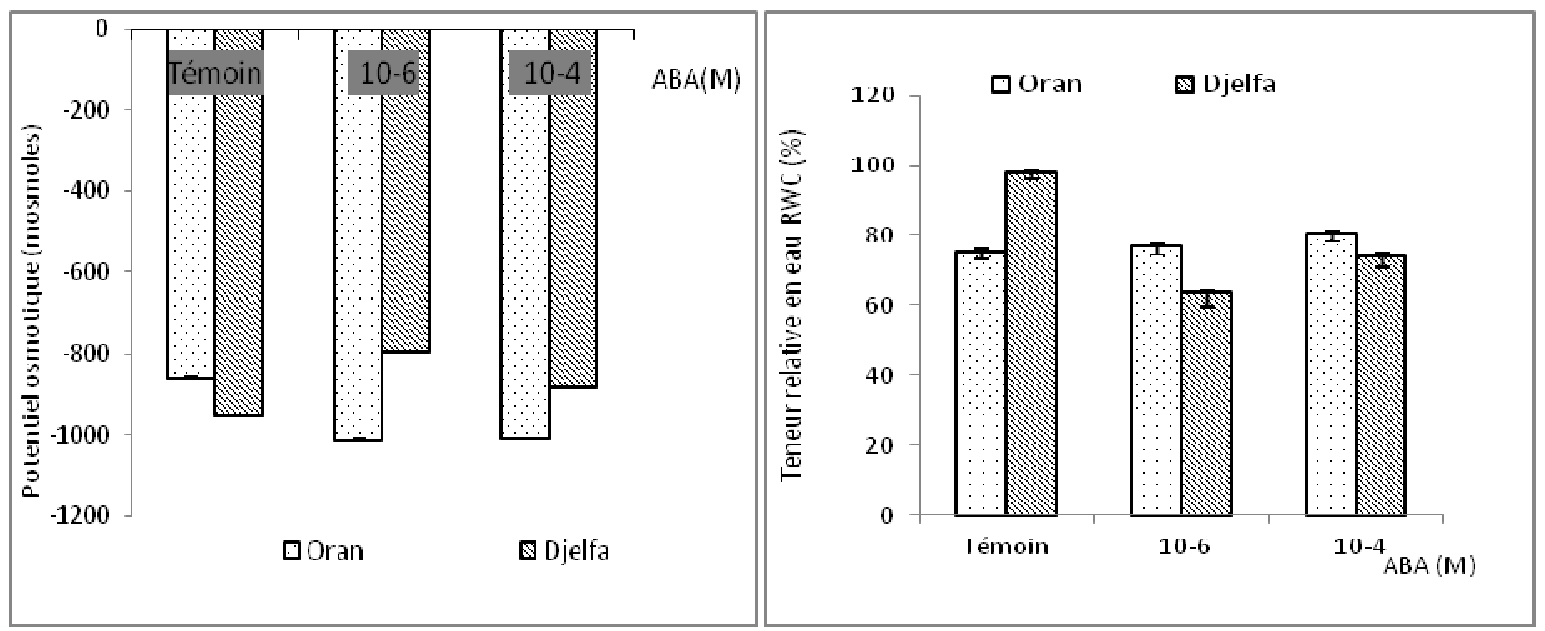

Fig.3. Evaluation du potentiel osmotique (à gauche) et de la teneur relative en eau (à droite) des feuilles de deux écotypes (Oran et Djelfa) après 21 jours de traitement à 
L'analyse statistique affiche des valeurs de $(\mathrm{F}=1353,523 ; \mathrm{p}=0,000$ à $\alpha<0.05)$ et $(\mathrm{F}=88,671$; $\mathrm{p}=0,000$ à $\alpha<0.05$ ) respectivement pour le $\Psi$ o et la RWC (Tableau 3).

Le Yo est inversement proportionnel à la RWC. Ceci est traduit statistiquement (Tableau 4) par l'existence d'une corrélation négative hautement significative entre les deux variables $(\mathrm{r}=$ $0,582, \mathrm{p}<0.001)$.

L'écotype d'Oran subit une augmentation du $\Psi$ o dés que les plantes reçoivent de l'ABA. Les valeurs affichées varient entre (-861,32 mosmoles) pour le témoin à $(-1014,12$ mosmoles et 1009,29 mosmoles) respectivement sous les traitements $10^{-6} \mathrm{M}$ et $10^{-4} \mathrm{M}$ d'ABA. L'écotype de Djelfa ne semble pas s'exprimer de la même manière que celui décrit précédemment. Les plantes témoins, cultivées sans apport de l'ABA, se caractérisent par des valeurs très négatives du potentiel osmotique (-953,16 mosmoles) qui se traduisent par la mise en œuvre d'un ajustement osmotique suite à une augmentation des pertes d'eau due à une faible résistance stomatique. La relation entre la résistance stomatique et le potentiel osmotique reste linéaire $(\mathrm{r}=0.460, \mathrm{p}<0.011)($ Tableau 4).

Par ailleurs, les résultats dégagés de l'analyse (Tableau 3) révèlent que les variations du Чo dépendent fortement de l'effet génotype $(\mathrm{F}=13606,913 ; \mathrm{p}=0,000$ à $\alpha<0.05)$ et de l'effet de l'interaction " ABA x écotype" ( $\mathrm{F}=16171,483 ; \mathrm{p}=0,000$ à $\alpha<0.05)$.

La RWC varie d'une manière très marquée sous le traitement hormonal $(F=88,671 ; p=0,000$ à $\alpha<0.05)$ et sous l'interaction " ABA x écotype" ( $F=133,796 ; \mathrm{p}=0,000$ à $\alpha<0.05)$. Par contre il n'y a pas d'effet génotype ( $\mathrm{F}=0,945 ; \mathrm{p}=0,000$ à $\alpha>0.05)$ (Tableau 3).

Pour l'écotype d'Oran, la RWC varie avec des valeurs très proches aussi bien pour les feuilles des plantes témoins que pour celles traitées à l'ABA aux deux concentrations. Au contraire, la réponse de l'écotype de Djelfa exprime des différences dans les variations de la RWC sous le traitement hormonal. En effet, les feuilles du témoin manifestent une RWC de $97.75 \%$; ce taux baisse jusqu'à $63,57 \%$ sous $10^{-6} \mathrm{M}$ ABA pour reprendre vers $73.98 \%$ sous le traitement à $10^{-4} \mathrm{M} \mathrm{ABA}$.

\subsection{Teneur des pigments chlorophylliens}

Les résultats, illustrés par la Figure 4, montrent que les teneurs en chl a et chl b varient avec l'écotype et le traitement hormonal. L'analyse statistique montre un effet significatif de l'ABA et du génotype sur les variations d'accumulation des pigments photosynthétiques. L'analyse 
décèle également une source de variation dans l'interaction " ABA x écotype" (Tableau 3).

Les valeurs des teneurs en chla et chl b sont plus élevées pour l'écotype d'Oran quel que soit le traitement à l'ABA alors que pour l'écotype de Djelfa, les deux types de chlorophylles subissent une chute significative notamment sous l'ABA à $10^{-4} \mathrm{M}$. La chlorophylle b s'est montrée plus sensible aux traitements ABA que la chl a $(r=0,448, p<0.013)$. Une relation de corrélation négative est observée entre le $\Psi$ o et la chl a $(\mathrm{r}=0,550, \mathrm{p}<0.002)$. Cette corrélation est traduite par des teneurs élevées en chl a en situation d'absence de toute variation de la turgescence (Tableau 4).

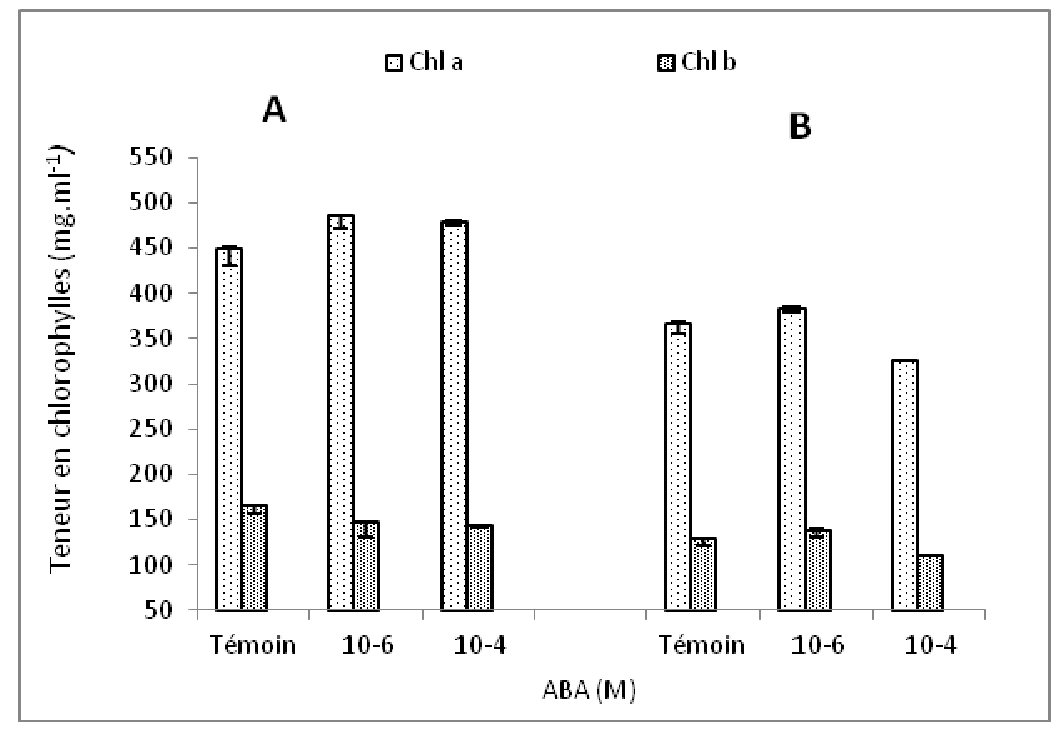

Fig.4. Variation des teneurs en chlorophylle a et en chlorophylle b des feuilles de deux écotypes, Oran (A) et Djelfa (B) après 21 jours de traitement à l'acide abscissique exogène

\section{DISCUSSION}

Les résultats que nous venons de décrire montrent que l'ABA intervient sur le comportement des plantes des deux écotypes d'Atriplex halimus (L.). En effet, il est rapporté par de nombreux travaux que cette hormone agit en conditions stressantes sur la fermeture des stomates [26, 27]. Cette réaction de la plante reste une composante majeure des mécanismes de la tolérance à la sécheresse [28]. Dans nos conditions expérimentales, les résultats indiquent d'abord que l'ABA exogène a un effet significatif sur la perte d'eau par 
transpiration (RWL) des feuilles des deux écotypes d'Atriplex. Les observations concluent que la transpiration diminue au cours du temps. Ceci présume que le délai de fermeture stomatique est d'autant plus court que la concentration en $\mathrm{ABA}$ est plus élevée ce qui est déjà rapporté par [29] sur le riz (Ovyza sativa $\mathrm{L}$.); à $10^{-4} \mathrm{M}$ d'ABA, la transpiration représente $40 \%$ de la valeur initiale et $90 \%$ à $10^{-6} \mathrm{M}$ ABA. Par ailleurs, la référence [30] a montré que des applications de l'ABA exogène à des concentrations comprises entre $10^{-3} \mathrm{M}$ et $10^{-4} \mathrm{M}$, ont provoqué la fermeture totale des stomates des feuilles de nombreuses espèces cultivées dans des conditions d'arrosages continus. D'autre part, les travaux de [31] sur les feuilles de Tradescantia virginiana et de [32] sur Arabidopsis thaliana ont mis en évidence l'ouverture stomatique associée à l'abaissement du niveau de l'ABA. Le comportement transpiratoire des plantes des deux écotypes d'Atriplex, varie avec l'écotype et la concentration en ABA. En effet, les plantes de l'écotype de Djelfa manifestent une réaction rapide à l'ABA traduite par une réduction des pertes d'eau par transpiration suite à la fermeture des stomates. D'autre part, la résistance stomatique, expression du degré de fermeture des stomates, montre des valeurs élevées chez le même écotype. Par contre, les résultats révèlent par ailleurs que les plantes de l'écotype d'Oran semblent réagir de manière modérée vis-à-vis de l'action de l'ABA, si bien que les stomates n'expriment que faiblement leur résistance jusqu'à un seuil critique de l'ABA $\left(10^{-4} \mathrm{M}\right)$ observé chez l'écotype de Djelfa. Le maintien des stomates ouverts malgré la contrainte prolongée implique que la feuille de l'écotype d'Oran peut transpirer sans limitations et que l'eau perdue est récupérée par un système racinaire très développé, puisque le contenu relatif en eau foliaire se maintient très élevé, associé à un potentiel osmotique bas $[33,9]$. La régulation stomatique rapide, révélée chez l'écotype de Djelfa, peut être une réponse adaptative à la sécheresse, permettant à la plante d'économiser l'eau disponible et de maintenir une teneur élevée en eau des tissus. Au contraire une fermeture stomatique plus lente, observée chez l'écotype d'Oran, peut traduire une certaine tolérance à la déshydratation qui peut être accompagnée d'un ajustement osmotique permettant de maintenir un niveau de turgescence élevé [34, 35, 36]. D’autres auteurs [37] ont prouvé que la résistance stomatique est significativement contrôlée par le potentiel hydrique. Les travaux de $[38,39,40,41]$ montrent que la sensibilité des stomates à l'ABA varie selon les espèces et d'autres paramètres en particulier le statut hydrique de la feuille. Les résultats obtenus sur la 
teneur relative en eau révèlent qu'elle est dépendante du traitement $\mathrm{ABA}$ mais pas de la variabilité génotypique. Pour l'écotype d'Oran, cette caractéristique augmente graduellement au cours du développement du stress à l'ABA, comparativement à l'écotype de Djelfa où la RWC a été sensiblement plus faible sous le traitement hormonal. Une telle réduction du RWC a été reportée chez de nombreuses espèces comme l'olivier et le gombo sous contrainte saline [42, 43] et chez l'Atriplex halimus exposée à un stress hydrique [44]. Les mêmes auteurs rapportent, par ailleurs, que l'addition de l'ABA au milieu de culture augmente le RWC des feuilles comparée à celle des non traitées [44]. Au cours de l'essai, les plantes témoins de l'écotype de Djelfa ont connu une augmentation des valeurs de la RWC et du Чo. Cela peut s'expliquer par la mise en œuvre d'un ajustement osmotique permettant de maintenir la turgescence cellulaire suite à une augmentation des pertes d'eau due à la réduction de la résistance stomatique [22].

Une forte résistance stomatique est généralement proposée comme critère d'adaptation à la sécheresse [45]. Il est admis que la fermeture stomatique réduit l'assimilation du $\mathrm{CO}_{2}$ et conduit inévitablement à une réduction de l'activité photosynthétique [46, 47]. Nos résultats concluent que les teneurs en chl a et chl b varient avec l'écotype et le traitement en ABA. En effet, ces valeurs sont plus élevées pour l'écotype d'Oran quel que soit le traitement à l'ABA alors que pour l'écotype de Djelfa, les deux types de chlorophylles subissent une chute significative notamment sous 1 ' $\mathrm{ABA}$ à $10^{-4} \mathrm{M}$. Il a été déjà rapporté que l'accroissement des concentrations en ABA ne cause aucun dommage aux mécanismes photosynthétiques et la baisse de la photosynthèse qu'il provoque peut être attribuée en partie à la fermeture des stomates limitant la quantité de $\mathrm{CO}_{2}$ entrant dans les tissus du mésophylle [48]. Les résultats obtenus lors de cette recherche indiquent l'existence des corrélations négatives entre la résistance stomatique et les chlorophylles $\mathrm{a}(\mathrm{r}=0,863)$ et $\mathrm{b}(\mathrm{r}=0,812)$. D'après la référence [49], la photosynthèse est hautement corrélée à la transpiration et à la conductance stomatique. Les auteurs de la référence [50] ont rapporté qu'une application quotidienne de $3.10^{-3} \mathrm{M}$ d'ABA à la surface des feuilles de Tradescantia virginiana et dans des conditions d'arrosages convenables, conduit à une réduction de la taille des stomates et de la conductance stomatique; alors que les mêmes auteurs signalent que la capacité photosynthétique n'est pas affectée. Il a été démontré aussi que la distribution inégale des 
stomates, induite par l'application de l'ABA, n'a pas d'incidence sur l'activité photosynthétique [51, 52]. Les auteurs de la référence [53], notent que l'ABA exogène améliore le rendement de la photosynthèse, la conductance stomatique et le taux de la transpiration en cas de sécheresse, avec une expression accrue des gènes sensibles à la sécheresse chez le riz. Selon la référence [47], la synthèse chlorophyllienne peut être réduite ou inhibée lors d'un stress hydrique.

\section{CONCLUSION}

Le comportement des écotypes d'Atriplex halimus L. analysés, varie en fonction des paramètres étudiés et du traitement hormonal appliqué. Il en résulte que l'écotype d'Oran s'est montré moins sensible à l'ABA que celui de Djelfa. Ce comportement de l'écotype d'Oran se traduit par une faible résistance stomatique qui peut être accompagnée d'un ajustement osmotique, ce dernier lui permettant d'éviter la déshydratation et d'accomplir sa photosynthèse.

Ainsi ce travail aura permis, grâce à quelques explorations fines, traduites en termes de réponses adaptatives, de sélectionner des traits qui peuvent être inclus dans un programme d'amélioration génétique pour la tolérance au stress hydrique.

\section{REFERENCES}

[1] Guo X S, Zhang W W, Huang Z Y J. Plant Ecol. 3, 2010, 79-87, doi: 10.1093/jpe/rtq007.

[2] Zhang W W, Yang H Q, You S Z, Fan S L, Ran K. Physiol. Biochem. 96, 2015, 254-260, doi: 10.1016/j.plaphy.2015.08.004.

[3] Showemimo F A, Olarewaju J D. Int. J. Plant Breed. Genet. 1, 2007, 29-33, doi: 10.3923/ijpbg.2007.29.33.

[4] Li C L, Wang M, Ma X Y, Zhang W. Mol. Plant. 7, 2014, 1508-1521, doi: $10.1093 / \mathrm{mp} / \mathrm{ssu} 061$.

[5] Levitt J, Responses of Plants to Environmental Stresses, Vol. II. Water, Radiation, Salt and Other Stresses. New York: Academic Press, 1980.

[6] De Parcevaux S., Huber L. Bioclimatologie: Concepts et applications. France: Eds. Quae, 2007, pp. $173-250$. 
[7] Seiler C, Harshavardhan V T, Rajesh K, Reddy P S, Strickert M, Rolletschek H, Scholz U, Wobus U, Sreenivasulu N. J. Exp. Bot. 62, 2011, 2615-2632, doi: 10.1093/jxb/erq446.

[8] Jammes F, Leonhardt N, Bousserouel D T , Very A A, Renou J P, Vavasseur A, Kwak J M, Sentenac H, Bouteau F, Leung J. Plant J. 79, 2014, 322-333, doi: $10.1111 /$ tpj.12564.

[9] Hirt H., Shinozaki K. Plant Responses to Abiotic Stress, Springer Science \& Business Media, 2004, pp.1-8.

[10] Rock C. D., Sakata Y., Quatrano R. S. Stress signaling I: the role of abscisic acid (ABA). In: A. Pareek, S. A. Sopory and H. J. Bohner Govindjee (Eds.), Abiotic stress adaptation in plants: physiological, molecular and genomic foundation, Springer, Dordrecht, 2010, pp.3373.

[11] Lianes A., Devinar G., Luna V. Role of abscisic acid in legumes under abiotic stress. In: M. A. Mohamed, A. Parvaiz (Eds.), Legumes under Environmental Stress: Yield, Improvement and Adaptations. John Wiley \& Sons, 2015, pp. 145-160.

[12] Annerose D., Cornaire B. Approche physiologique de l'adaptation à la sécheresse des espèces cultivées pour l'amélioration de la producton en zones sèches. In F. N. Reyniers and L. Netoyo (Eds.), Bilan hydrique agricole et sécheresse en Afrique tropicale. Paris: John Libbey Eurotext, 1994, pp. 137-150.

[13] Bingbing L., Wensuo J. ABA Transport and Distribution in Relation to Its Function in Plants. In: D.P. Zhang (Ed.), Abscisic Acid: Metabolism, Transport and Signaling. Netherlands: Springer, 2014, pp. 61-75.

[14] McCann S.E., Huang B. Evaluation of drought tolerance and avoidance traits for six creeping bentgrass cultivars. Hortscience, 2008, 43(2), 519-524.

[15] Kuromori T., Sugimoto E., Shinozaki K., Plant J. 2011, 67 (5), 885-94, doi: 10.1111/j.1365-313X.2011.04641.X.

[16] Leung J., Valon C., Moreau B., Boeglin M., Lefoulon C., Joshi-Saha A., Chérel I., Biologie Aujourd'hui, 2012, 206 (4), 301-312,

doi : 10.1051/jbio/2012029. 
[17] Paul R., Impens R. Les maladies non parasitaires, In: P. Lepoivre (Ed.), Phytopathologie: Bases moléculaires et biologiques des pathosystèmes et fondements des stratégies de lutte. France: De Boeck Supérieur, 2003, pp. 23-40.

[18] Brendel O., Cochard H. Comment les espèces végétales s'adaptent au stress hydrique. In: Y. Birot, C. Gracia, G. Matteucci, R. Mavsar, B. Muys \& M. Palahi (Eds.), L'eau pour les forêts et les hommes en Région Méditerranéenne: un équilibre à trouver. European Forest Institute, 2011, pp. 84-91.

[19] Johnson G. B., Raven P. H., Masson K. A., Losos J. B., Singer S.R. Biologie - Version luxe. France: De Boeck Supérieur, 2011, pp.825-837.

[20] Gallais A. Comprendre l'amélioration des plantes: Enjeux, méthodes, objectifs et critères de sélection. France: Quae, 2015, pp.147-159.

[21] Finkelstein R. Abscisic acid synthesis and response. Arabidopsis Book, 11, 2013. doi:10.1199/tab.0166.

[22] El Jaafari S, Paul R, Lepoivre P, Semal J, Laitat E. Résistance à la sécheresse et réponses à l'acide abscissique: analyse d'une approche synthétique. Cahiers Agricultures, 2, 1993, 256263.

[23] Clarck J M, Romagosa I, Jana S, Strivastava J P, and Mccaid T N. Relation of excised leaf water lose rate and yield of durum wheat in diverse environments. Can. J. Plant .Sci. 69, 1989, 1057-1081.

[24] Barrs H D, Weatherley P E. Aust. J. Sci. 15, 1968, 413-428, doi:10.1071/BI9620413.

[25] Arnon D. Coppere enzymes in isolated chloroplasts. Polyphenoloxydase in Beta vulgaris. Plant Physiol., 1949, 24(1), 1-15.

[26] Israelsson M, Siegel R S, Young J, Hashimoto M, Iba K, and Schroeder J.I. Guard cell ABA and $\mathrm{CO}_{2}$ signaling network updates and $\mathrm{Ca}^{2+}$ sensor priming hypothesis. Curr. Opin. Plant Biol., 2006, 9(6):654-663.

[27] Kuromori T., Sugimoto E., Shinozaki K., Plant J. 2011, 67(5), 885-94, doi: 10.1111/j.1365-313X.2011.04641.x. 
[28] Maury P, Langlade N, Grieu P, Rengel D, Sarrafi A, Debaeke P, and Vincourt P. Ecophysiologie et génétique de la tolérance à la sécheresse chez le tournesol. Innovations Agronomiques, 14, 2011, 123-138.

[29] Bois J F, Dizes J, and Lasceve G. Réponse du riz à l'application d'acide abscissique exogène: Fermeture stomatique et enroulement foliaire. C. R. Acad. Sci., 1987, 305(3): 449452.

[30] Hopkins W G. Physiologie végétale. France : De Boeck Supérieur, 2003, pp.309-335.

[31] Nejad A R, Van Meeteren U. The role of abscisic acid in disturbed stomata characteristics of Tradescantia Virginia during growth at high relative air humidity. J. Exp. Bot., 2007, 58(3), 627-636.

[32] Aliniaeifard S., Matamoros P.M., Meeteren U.V., Physiol. Plant. 2014,152 (4), 688-699, doi: 10.1111/ppl.12216.

[33] Chimenti CA, Pearson J, Hal AJ. Field Crops Res. 75, 2002, 235-246, doi: 10.1016/S0378-4290(02)00029-1.

[34] Rejeb M.N., Laffray D., Louguet P. Modification de la conductance stomatique de diverses origines tunisiennes de caroubier (Ceratonia siliqua L.) soumises à une contrainte hydrique prolongée. In: Y. Demarly and N. Chalbi (Eds.), L'amélioration des plantes pour l'adaptation aux milieux arides. France: John Libbey Eurotext, 1991, pp. 149-158.

[35] Abebe T, Guenzi A C, Martin B, and Cushman J C. Tolerance of mannitol accumulating transgenic wheat to water stress and salinity. Plant Physiol., 2003, 131(4):1748-1755.

[36] Maisura M A, Lubis C I, Junaedinand A, and Ehara H. Some physiological character responses of rice under drought conditions in a paddy system. J. Int. Soc. Southeast Asian. Agric. Sci., 2014, 20(1):104-114.

[37] Lu C, Zhang J. Effect of water stress on photosystem II photochemistry and its thermostability in wheat plants. J. Exp. Bot., 1999, 50(366), 1199-1206.

[38] Tardieu F, Simonneau T. J. Exp. Bot. 49, 1998, 419-432, doi: $10.1093 / \mathrm{jxb}$.

[39] Niinemets U, Sober A, Kull O, Hartung W, Tenhunen J D. Int. J. Plant Sci. 160, 1999, 707-721, doi: 10.1086/314173. 
[40] Augé RM, Green C D, Stodola A J, Saxton A M, Olinick J B, and Evans R M. Correlations of stomatal conductance with hydraulic and chemical factors in several deciduous tree species in a natural habitat. New Phytol., 2000, 145(3):483-500.

[41] Wilkinson S and Davies W J. ABA-based chemical signalling: the co-ordination of responses to stress in plants. Plant Cell Environ., 2002, 25(2), 195-210.

[42] Kasraoui M F, Denden M, Braham M, Garcia M, and Lamaze T. Comportement physiologique de deux variétés d'olivier (Olea europaea L.) soumises à une contrainte hydrique. Annales de l'INGREF, 6, 2004, 49-67.

[43] Achour A, Bidai Y, and Belkhodja M. L'impact de la salinité sur le comportement hydrique et métabolique d'une variété de Gombo Abelmoschus esculentus L.). International Journal of Innovation and Applied Studies, 2015, 12(4):943-953.

[44] Ben Hassine A, Ghanem M E, Bouzid S, Lutts S. Ann. Bot. 104, 2009, 925-936, doi: 10.1093/aob/mcp174.

[45] Turner N C. Aust. J. Plant Physiol.13, 1986, 175-190, doi:10.1071/PP9860175.

[46] Flexas J., Baron M., Bota J., Ducruet J.M., Galle A., Galmes J., Jimenez M., Pou A., Ribas-carbo M., Sajnani C., Tomas M., Medrano H. J. Exp. Bot. 2009, 60(8), 2361-2377, doi: $10.1093 / \mathrm{jxb} / \mathrm{erp} 069$.

[47] Pandey V., Shukla A., Rice Science, 2015, 22(4), 147-161, doi: 10.1016/j.rsci.2015.04.001.

[48] Cornic G. Effet de la contrainte hydrique sur la photosynthèse foliaire : De l'utilisation expérimental des relations A/Ci et ACc, 2008, article, $36 \mathrm{P}$.

[49] Natali S, Bignami C, and Fusari A. Water consumption, photosynthesis and water potential in Olea europea L., cv. Frantoio, at different levels of available water. Agriculture Méditerranéenne, 121, 1991, 205-212.

[50] Franks P J, Farquhar G D. Plant Physiol. 2001, 125(2), 935-942, doi:10.1104/pp.125.2.935.

[51] Terashima I, Wong S C, Osmond C B and Farquhar G D. Characterization of nonuniform photosynthesis induced by abscisic acid in leaves having different mesophyll anatomies. Plant Cell. Physiol. 29, 1988, 385-395. 
[52] Mott K A. Plant Cell. Environ. 1995, 18(11),1291-1300,

doi: 10.1111/j.1365-3040.1995.tb00188.x.

[53] Teng K.Q., Li J.Z., Liu L., Han Y.C., Du Y.X., Zhang J., Sun H.Z., Zhao Q.Z. Acta Physiol. Plant. 2014, 36(8), 2219-2227, doi:10.1007/s11738-014-1599-4.

\section{How to cite this article:}

Bidai Y, Achour A and Belkhodja M. Abscisic acid effects on water and photosynthetic characteristics of two ecotypes of Atriplex halimus L. J. Fundam. Appl. Sci., 2016, 8(2), 452469. 